\title{
Emerging azithromycin-resistance among the Neisseria gonorrhoeae strains isolated in Hungary
}

\author{
Alexandra Brunner ${ }^{1}$, Eva Nemes-Nikodem² ${ }^{2}$ Csaba Jeney $^{3}$, Dora Szabo ${ }^{3}$, Marta Marschalko ${ }^{1}$, Sarolta Karpati ${ }^{1}$ \\ and Eszter Ostorhazi ${ }^{3^{*}}$
}

\begin{abstract}
Background: In the 1990s, azithromycin became the drug of choice for many infectious diseases but emerging resistance to the drug has only been reported in the last decade. In the last 5 years, the National Neisseria gonorrhoeae Reference Laboratory of Hungary (NNGRLH) has also observed an increased number of $N$. gonorrhoeae strains resistant to azithromycin. The aim of this study was to determine the most frequent sequence types (ST) of N. gonorrhoeae related to elevated levels of azithromycin MIC (minimal inhibitory concentration). Previously and currently isolated azithromycin-resistant strains have been investigated for the existence of molecular relationship.
\end{abstract}

Methods: Maldi-Tof technic was applied for the identification of the strains isolated from outpatients attending the reference laboratory. Testing antibiotic susceptibility of azithromycin, cefixime, ceftriaxone, tetracycline, spectinomycin and ciprofloxacin was carried out for all the identified strains, using MIC strip test Liofilchem ${ }^{\circledR}$. N. gonorrhoeae multiantigen sequence typing (NG-MAST) was performed exclusively on azithromycin-resistant isolates. A phylogenetic tree was drawn using MEGA6 (Molecular Evolutionary Genetics Analysis Version 6.0) Neighbour-Joining method.

Results: Out of 192 N. gonorrhoeae isolates, $30.0 \%$ (58/192) proved resistant to azithromycin (MIC > 0.5 mg/L). Of the azithromycin-resistant isolates, ST1407, ST4995 and ST11064 were the most prevalent. Based on the phylogenetic analysis, the latter two STs are closely related.

Conclusions: In contrast to West-European countries, in our region, resistance to azithromycin has increased up to $30 \%$ in the last 5 years, so the recommendation of the European Guideline $-500 \mathrm{mg}$ of ceftriaxone combined with $2 \mathrm{~g}$ of azithromycin as first choice therapy against N. gonorrhoeae- should be seriously considered in case of Hungary.

Keywords: Azithromycin-resistance, Neisseria gonorrhoeae, Sequence types, Phylogenetic tree

\section{Background}

The treatment of gonorrhoea infection poses a continuous problem as Neisseria gonorrhoeae has developed resistance to each antimicrobials used in the past 70 years [1]. Therefore, it is necessary to enhance the surveillance of gonococcal antimicrobial resistance, especially for the drugs of first choice: ceftriaxone and azithromycin [2]. In Hungary, resistance to ceftriaxone has not yet

\footnotetext{
*Correspondence: ostorhazi.eszter@med.semmelweis-univ.hu

${ }^{3}$ Institute of Medical Microbiology, Semmelweis University, 4 Nagyvárad

Square, Budapest, Hungary

Full list of author information is available at the end of the article
}

been reported. In contrast, the appearance and spread of azithromycin-resistance have been observed in the last 4 years [3].

Since the 1990s, azithromycin has become the drug of choice for many infections, such as sexually transmitted diseases (STDs), community-acquired pneumonia, acute bacterial sinusitis, otitis media, tonsillitis, pharyngitis, skin infections or acute bacterial exacerbations of chronic obstructive pulmonary disease [4]. Of STDs, azithromycin is used to treat uncomplicated gonorrhoea in patients with cephalosporin allergy, Chlamydia trachomatis coinfection, Heamophilus ducreyii, Ureaplasma urealyticum, Mycoplasma genitalium infections. This antibiotic 
revolutionised the therapy as it shortened treatment time from 7-14 days to 1-5 days and improved patient compliance due to high tissue levels and long half-life. New administration formulations such as sustained-release microspheres allowed higher doses to be administered and reduced gastrointestinal side-effects, so azithromycin seemed to be capable of approaching the concept of an ideal antibiotic [5]. However, recently decreased antimicrobial susceptibility to azithromycin may disprove this assumption.

According to the data of the European Surveillance of Antimicrobial Consumption (ESAC), in Hungary the outpatient consumption of antimicrobials was 16.0 defined daily doses (DDD) per 1000 inhabitants per day. This number can be subdivided into major antibiotic classes such as penicillins topping the list by DDD of 7.19, macrolides taking the second place with DDD of 2.94 and, finally, cephalosporins with DDD of 2.13 [6].

Nevertheless, at the National Neisseria gonorrhoeae Reference Laboratory of Hungary (NNGRLH), we observed the appearance and rapid spread of azithromycin-resistance in Hungary between 2010 and 2013. We aimed to survey the antimicrobial susceptibility in 2014 and compare it with the data of the last 4 years and characterise the azithromycin-resistant strains by NG-MAST.

Molecular evolutionary analysis was conducted and genetic relationships were estimated between the STs spreading in Hungary in 2014.

\section{Methods}

\section{Bacterial strains and medical records}

The NNGRLH at the STD Centre in the Department of Dermatology, Venerology and Dermatooncology of Semmelweis University, Budapest, Hungary collected samples from consecutive symptomatic gonorrhoea patients and from their asymptomatic contacts in 2014. The samples were cultured, characterised and stored on Cryobank breads (Mast Diagnostic, Germany) at $-80{ }^{\circ} \mathrm{C}$. Clinical data such as sex, age, sexual orientation, anatomic site of infection were recorded. C. trachomatis co-infection was also screened. Patients' data were analysed according to law 1997/CLIV 26\$ taking into account maximum privacy rights and anonymity of patients [7].

\section{Antibiotic susceptibility}

Clinical samples -cervical, anal, urethral and pharyngeal swabs- were obtained and grown on preheated VCA3 agar (Biomérieux, Budapest, Hungary) and on non-selective PVX chocolate agar (Biomérieux, Budapest, Hungary) at $37^{\circ} \mathrm{C}$ in an atmosphere of $5 \%$ of carbon dioxide for 24-48 h. Minimum inhibitory concentrations (MIC; $\mathrm{mg} / \mathrm{L}$ ) were determined for azithromycin, cefixime, ceftriaxone, tetracycline, spectinomycin and ciprofloxacin on PVX chocolate agar (Biomérieux, Budapest, Hungary) using MIC strip tests (Liofilchem ${ }^{\circledR}$ s.r.l., Roseto degli Abruzzi, Italy) according to the manufacturer's instructions, using a direct colony suspension equivalent to McFarland standard of 0.5 . Testing conditions also included incubation at $36.5{ }^{\circ} \mathrm{C}$ and $5 \%$ of carbon dioxide for $24 \mathrm{~h}$. All results were interpreted by using breakpoints for susceptibility and resistance according to the European Committee on Antimicrobial Susceptibility Testing (EUCAST) [8]. Concerning the MIC breakpoints of azithromycin, strains with MICs over $0.25 \mathrm{mg} / \mathrm{L}$ but below $0.5 \mathrm{mg} / \mathrm{L}$ were considered to be of intermediate resistance. Isolates with MICs higher than $0.5 \mathrm{mg} / \mathrm{L}$ were considered resistant. N. gonorrhoeae ATCC 49226, with an azithromycin MIC of $0.12 \mathrm{mg} / \mathrm{L}$, was used as a control strain to ensure the quality of the susceptibility tests.

\section{Molecular methods}

Out of the 58 resistant and 42 intermediately resistant strains 29 and 21 were selected for $N$. gonorrhoeae multiantigen sequence typing (NG-MAST) according to a previously described method [9]. The sequences of por $B$ and $t b p B$ PCR products were determined after their preliminary purification by the Exosap IT purification kit (Affymetrix, USA). BigDye ${ }^{\circledR}$ Terminator v3.1 Cycle Sequencing Kit (Life Technologies, USA) was used and the same forward and reverse primers were applied as for porB and $t b p B$ PCR methods. Last purification was carried out by NucleoSEQ Column PCR Purification Kit (Macherey-Nagel, Germany). Nucleotide sequences were determined by capillary electrophoresis, with a capillary length of $50 \mathrm{~cm}$ and POP-7 polymer on ABI 3130xl Genetic Analyzer (Applied Biosystems, Foster City, CA, USA).

NG-MAST STs including the new alleles and STs were assigned on the NG-MAST website (www.ng-mast.net).

Phylogenetic tree was constructed by MEGA6 (Molecular Evolutionary Genetics Analysis Version 6.0) Neighbour-Joining algorithm, using maximum composite likelihood model [10]. The degree of similarity was determined using the highly similar sequence (Megablast) BLASTN Program of the National Library of Medicine of the National Center for Biotechnology Information [11].

\section{Results}

In 2014, 192 N. gonorrhoeae strains were isolated at the STD Centre of the Department of Dermatology, Venerology and Dermatooncology of Semmelweis University, Budapest, Hungary. The number of patients attending our STD centre makes up about $10 \%$ of the total number of notified gonorrhoea infections in Hungary year by year. However, the ratio of $N$. gonorrhoeae positive patients to total patients examined increased from $7.8 \%$ 
in 2013 to $10.85 \%$ by the end of 2014 . Of the 192 N. gonorrhoeae, $85 \%$ were isolated from male patients (median age 32 years); the remaining strains were collected from females (median age 26 years).

Urethritis was found in $77.3 \%$ of male patients, while in females the dominant anatomical site of infection was the urethra $(68.9 \%)$ and cervix $(65.5 \%)$. Symptomatic infections or asymptomatic carrier states were detected in the anus $(20.2 \% / 44.8 \%)$ and in the pharynx $(17.9 \% / 24.1 \%)$ in male/female patients, respectively.

All the 192 isolates were susceptible to ceftriaxone and spectinomycin. The prevalence of ciprofloxacin and tetracycline resistance -39.8 and $70 \%$, respectivelyremained as high as in previous years in Hungary. However, the MIC averages of ceftriaxone and cefixime have increased in the last few years. Cefixime MIC exceeded the resistant breakpoints in $1.57 \%$ of the strains.

Of the 192 strains, $92(48 \%)$ were susceptible to azithromycin and 100 (52\%) exhibited reduced susceptibility. Fifty-eight of these 100 strains $-30.0 \%$ of all the strains-were resistant to azithromycin, according to the breakpoints of EUCAST. The percentage of azithromycin resistance showed a significant increase from $15.9 \%$ in 2013 to $30.0 \%$ in 2014 ( $\chi^{2}=11.4437, P$ value is 0.000717 , $P<0.001)$. Concerning the strains with reduced susceptibility to azithromycin, we can say that the ratio of female/ male patients was $1-7.3$. MICs of $\geq 1 \mathrm{mg} / \mathrm{L}$ for azithromycin were observed in $7.0 \%(13 / 192)$ of the isolated $N$. gonorrhoeae strains out of which three had an MIC of $1.5 \mathrm{mg} / \mathrm{L}$ (Additional file 1: Figure S1).

The prevalence of $C$. trachomatis infection detected by multiplex RT-PCR was only $6.7 \%$ in 2014 at the NNGRLH, but $N$. gonorrhoeae positivity was found in $12.2 \%$ of cases. Only $14.7 \%$ of the gonorrhoea-positive samples were co-infected with $C$. trachomatis.

The $50 \mathrm{~N}$. gonorrhoeae resistant or intermediateresistant isolates to azithromycin were divided into 34 NG-MAST sequence types, and a unique NG-MAST sequence type was found for 10 isolates. The three dominant strains were ST1407, ST4995 and ST11064, each represented by 5 isolates (10-10\%). Regarding frequency, these were followed by ST 4417 represented by 3 isolates, then by ST 995 and ST 8517, each represented by 2 isolates. The 29 other STs were represented by only one isolate. Ten new STs, which had not been previously described in the world, were assigned as ST 11699 to ST 11708 on NG-MAST website. Four of them, ST 11703, 11706-11708, due to new allele combinations of known por $B$ and $t b p B$ alleles, were assigned on the website. The other 6 new STs had new porB or $\operatorname{tbp} B$ alleles (Table 1 ).

According to the phylogenic tree in Fig. 1, the azithromycin-resistant and intermediately resistant strains isolated in NNGLRH in 2014 were divided into three major
Table 1 Incidence of sequence types, por $B$ and tbpB allels among azithromycin-resistant or intermediate-resistant $N$. gonorrhoeae strains isolated in Hungary in 2014

\begin{tabular}{|c|c|c|c|c|c|}
\hline \multirow[t]{2}{*}{ ST } & \multirow[t]{2}{*}{ porB allele } & \multirow[t]{2}{*}{ tbpB allele } & \multirow[t]{2}{*}{$\begin{array}{l}\text { Number } \\
\text { of strains }\end{array}$} & \multicolumn{2}{|c|}{$\begin{array}{l}\text { Azithromycin } \\
\text { susceptibility } \\
\text { category, number } \\
\text { of strains }\end{array}$} \\
\hline & & & & 1 & $\mathbf{R}$ \\
\hline 10081 & 5921 & 29 & 1 & 1 & - \\
\hline 10083 & 3031 & 29 & 1 & 1 & - \\
\hline 995 & 28 & 29 & 2 & - & 2 \\
\hline 4417 & 2707 & 894 & 3 & 1 & 2 \\
\hline 10087 & 35 & 29 & 1 & - & 1 \\
\hline 5333 & 3229 & 137 & 1 & - & 1 \\
\hline 225 & 4 & 4 & 1 & - & 1 \\
\hline 10088 & 2700 & 4 & 1 & 1 & - \\
\hline 11706 & 1183 & 1388 & 1 & - & 1 \\
\hline 7232 & 1489 & 1388 & 1 & - & 1 \\
\hline 11702 & 6870 & 1582 & 1 & 1 & - \\
\hline 8706 & 35 & 1582 & 1 & 1 & - \\
\hline 11704 & 6871 & 2003 & 1 & - & 1 \\
\hline 11708 & 1183 & 18 & 1 & - & 1 \\
\hline 8465 & 4864 & 18 & 1 & - & 1 \\
\hline 2400 & 1489 & 563 & 1 & - & 1 \\
\hline 8115 & 3942 & 563 & 1 & - & 1 \\
\hline 11707 & 4864 & 563 & 1 & - & 1 \\
\hline 10101 & 4160 & 110 & 1 & 1 & - \\
\hline 8517 & 1142 & 1531 & 2 & 1 & 1 \\
\hline 3378 & 2043 & 110 & 1 & 1 & - \\
\hline 8826 & 5213 & 110 & 1 & - & 1 \\
\hline 1407 & 908 & 110 & 5 & 2 & 3 \\
\hline 11699 & 6867 & 138 & 1 & 1 & - \\
\hline 21 & 14 & 33 & 1 & - & 1 \\
\hline 11064 & 14 & 1131 & 5 & 2 & 3 \\
\hline 11703 & 1582 & 1131 & 1 & 1 & - \\
\hline 10593 & 581 & 1131 & 1 & 1 & - \\
\hline 11700 & 6868 & 1131 & 1 & 1 & - \\
\hline 4995 & 3031 & 33 & 5 & 3 & 2 \\
\hline 5343 & 6195 & 1131 & 1 & - & 1 \\
\hline 11337 & 6630 & 1131 & 1 & - & 1 \\
\hline 11705 & 6872 & 2004 & 1 & 1 & - \\
\hline 11701 & 6869 & 1131 & 1 & - & 1 \\
\hline$\sum=$ & & & 50 & 21 & 29 \\
\hline
\end{tabular}

groups based on closer relationship. From the most prevalent STs, the first group contained ST225; ST1407 belonged to the second group, whereas the third group contained ST4995 and ST11064. A similarity of at least $96 \%$ can be shown for all members of the third group. The biggest similarity-99 \%- was detected between ST21, ST11064 and ST11703. A 98 \% similarity between 


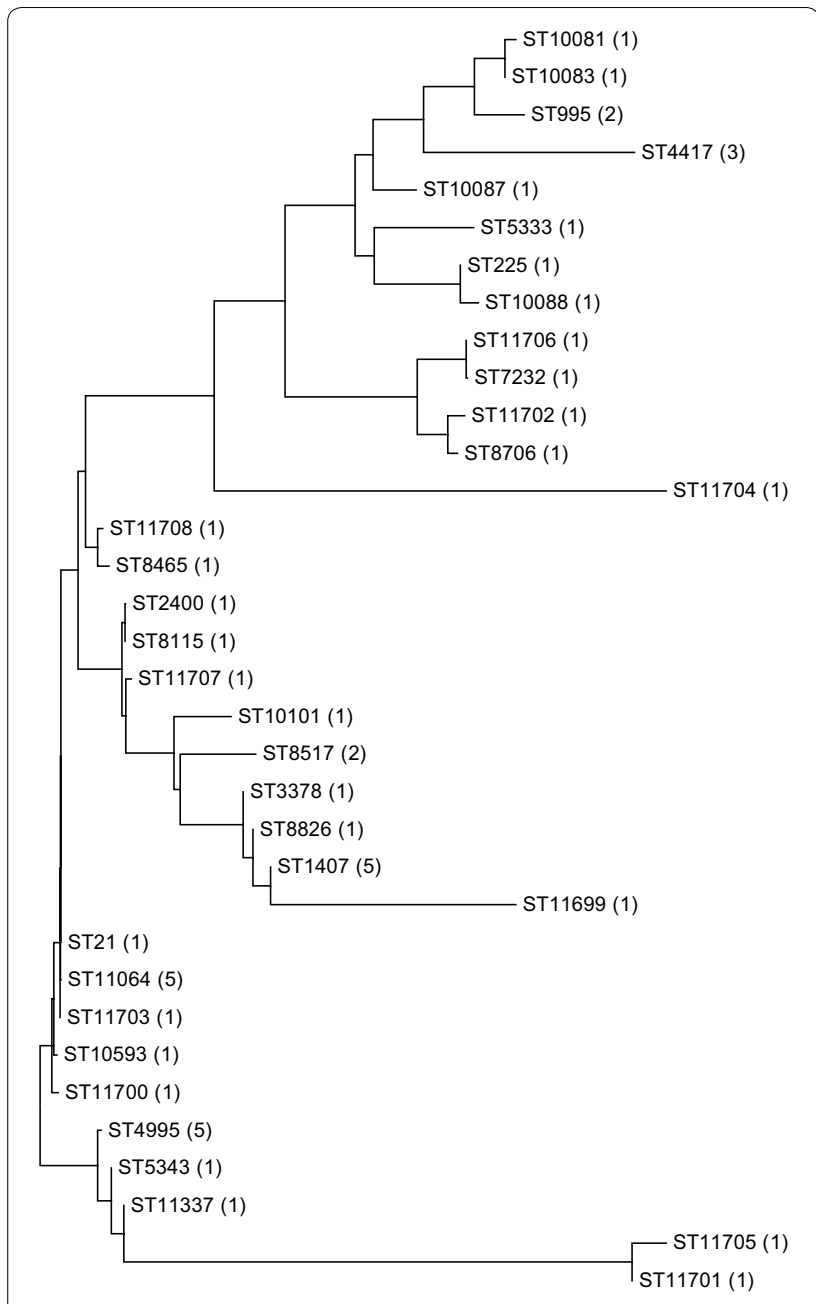

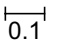

Fig. 1 Phylogenic tree constructed from porB and tbpB allels (ST) from 50 azithromycin-resistant or intermediate-resistant strains isolated in 2014 in Hungary. Number of isolates according to the appropriate STs is indicated in parenthesis

ST11703 and ST10593, or between ST10593 and ST11700 was detected, but ST11700 and ST11703 demonstrated only a $97 \%$ similarity. With the latter two STs, ST4995 showed a similarity of $96 \%$. Between ST 4995 and ST5343 or ST11337 a similarity of $97 \%$ was detected.

\section{Discussion}

For the treatment of gonorrhoea, the European Guideline, published in 2012, recommends $500 \mathrm{mg}$ of ceftriaxone combined with $2 \mathrm{~g}$ of azithromycin as first choice therapy [2]. This dual therapy is supported by European epidemiological surveys, mostly based on western European data. These European epidemiological surveys demonstrate that $C$. trachomatis co-infection is so common in young heterosexual patients under 30 and in MSM with gonorrhoea that $1 \mathrm{~g}$ of azithromycin administered orally as a single dose or $100 \mathrm{mg}$ of doxycycline administered orally twice daily for 7 days should be given unless co-infection has been excluded by NAAT testing.

Since resistance to azithromycin increased from 0 to $30 \%$ in Hungary in the last 5 years, and WHO recommended [1] that an antimicrobial should not be used when $>5 \%$ of the strains are resistant, we aimed to conduct an active surveillance to detect recent emergence in Hungary. Our theory for the increasing azithromycin resistance is that, according to ESAC, azithromycin has become the second most commonly used antimicrobial in Hungary. Hence, patients might have been treated previously with azithromycin for an infection, microbiome in the pharynx or anus, could have been exposed to this antibiotic several times, could have acquired resistance and acted as reservoirs of genes [12]. Azithromycin-resistant samples were frequently isolated from the pharynx (21\%) or the anus (32.5\%), which may refer to the fact that the asymptomatic carrier state provides an opportunity for commensal microbiome and $N$. gonorrhoeae to exchange their resistance genes, resulting in a hyperexpression of the efflux pump MtrCDE or mutation in the $23 \mathrm{~S}$ rRNA $[13,14]$. The high percentage of infections in the pharynx and anus do not only involve the risk of acquiring resistance, but also the risk of developing a disseminated infection and the spread of asymptomatic gonorrhoea.

Previously, the most frequent isolated STs were ST2992, ST1407, ST4995 and ST225 in Hungary [15]. In our recent study, three groups of STs based on closer relationship, were observed among azithromycin-resistant or intermediate-resistant $N$. gonorrhoeae strains isolated in Hungary in 2014. The first group contains the previously described ST225; ST1407 belongs to the second group, while ST4995 and ST11064 are included in the third group (Fig. 1). The latter two STs represent the $20 \%$ of the azithromycin-resistant strains but, according to the data of Table 1 and Fig. 1, it can be presumed that more than $30 \%$ of the azithromycin-resistant isolates are closely related in the third group of STs (similarity is at least $96 \%$ ). The previously described uniquely high prevalence of ST4995 in Hungary confirms the theory that the isolates of the third neighbourhood group are successful strains in this country, and may cause therapeutic failure in our region in the future. Nevertheless, based on the exact definition of genogroup-one identical allele is shared and the other allele shows a similarity of $\geq 99 \%$ [16] - only ST21, ST11064 and ST11703 make up a genogroup. This genogroup is named G11064, since ST11064 is the predominant ST within the group.

The other prevalently isolated strains in the second group of neighbourhood are associated with ST1407, i.e. 
the ST previously described as resistant to ciprofloxacin, tetracycline and strongly associated with decreased susceptibility to cephalosporins $[16,17]$. Poorly controlled use of antibiotics may promote the selection and spread of multidrug-resistant strains from this group [18].

The rapid selection of azithromycin-resistant strains in Hungary shows that azithromycin might not be optimised for the treatment of gonorrhoea, neither in monotherapy nor in dual therapy. On the one hand, the question is whether the combination of cephalosporins with azithromycin decreases the MICs of cephalosporins or not. There are some studies which report in vitro synergy between third-generation cephalosporins and azithromycin [19], but others do not [20]. The clinical efficacy of the dual therapy with ceftriaxone and azithromycin could be lower than ceftriaxone monotherapy since the combination was less bactericidal as ceftriaxone alone in a time-kill experiment [21].

On the other hand, we have to investigate the prevalence of $C$. trachomatis co-infection. In Europe, according to ECDC's data, Chlamydia is the most frequently reported STD. In 2011, 346911 cases were notified in 25 European countries, and 39179 cases of gonorrhoea were registered. By contrast, in our laboratory twice as many gonorrhoea infections were identified as Chlamydia infections; $6.7 \%$ of the samples of the STD centre were Chlamydia-positive and $12.2 \%$ were gonorrhoea-positive. These data correlate with those of the National Epidemiological Laboratory, where 1077 cases of Chlamydia and 1525 cases of gonorrhoea were recorded in 2013. While the surveys of IUSTI refer to the common prevalence of co-infection, in our laboratory only $14.7 \%$ of the gonorrhoea-positive samples were co-infected with $C$. trachomatis. This also raises the question whether combination therapy is the appropriate treatment in Hungary for gonorrhoea infection. Furthermore, as the increasing azithromycin-resistance and also the emerging cefixime and ceftriaxone MICs threaten the currently recommended therapies for gonorrhoea, it would be essential to replace these antimicrobials with novel ones which have not been used before for gonorrhoea infection in our country. The use of solithromycin, gentamicin, gemifloxacin might be a short term solution, but developing novel antimicrobials is essential [22, 23].

\section{Conclusions}

In Hungary, the treatment of gonorrhoea infections relies on the combination therapy of ceftriaxone and azithromycin, recommended by the international guidelines. However, the data of this study should not only draw attention to caution in the use of azithromycin as the sole treatment for gonorrhoea but also to that in the use of combination therapy in Hungary. This study indicates that the rate of azithromycin-resistant strains is growing in our country year by year. We observed a unique emergence of azithromycin-resistant $N$. gonorrhoeae strains the amount of which has doubled in a period of 1e year. Besides, the strains being the most common in Hungary in 2013, also appeared in 2014 and were associated with azithromycin-resistance. This experience restricts the usefulness of this antibiotic recommended as first-line treatment for gonorrhoea worldwide and argues for regular surveillance to determine azithromycin-susceptibility.

\section{Additional file}

Additional file 1: Figure S1. Distribution of azithromycin MIC among N. gonorrhoeae strains in 2014.

\section{Abbreviations}

ECDC: European Centre for Disease Prevention and Control; ESAC: European Surveillance of Antimicrobial Consumption; EUCAST: European Committee on Antimicrobial Susceptibility Testing; DDD: defined daily doses; IUSTI: International Union against Sexually Transmitted Infections; NG-MAST: N. gonorrhoeae multiantigen sequence typing; MEGA6: Molecular Evolutionary Genetics Analysis Version 6.0; MIC: minimal inhibitory concentration; MSM: men who have sex with men; NNGRL: National Neisseria gonorrhoeae Reference Laboratory of Hungary; ST: sequence type; STD: sexually transmitted diseases.

\section{Authors' contributions}

BA, OE wrote the manuscript. BA, NNE conducted the review of the literature relevant to the paper. MM and SK examined and treated the patients. NNE and OE performed identification and susceptibility testing of the strains. BA, JCS and SZD made the molecular examinations of the strains and prepared the phylogenetic tree. Each author contributed suggestions to the manuscript. All authors read and approved the final manuscript.

\section{Author details \\ ${ }^{1}$ Department of Dermatology, Venerology and Dermatooncology, Semmel- weis University, 41 Mária Street, Budapest, Hungary. ${ }^{2}$ Department of Labora- tory Medicine, Semmelweis University, 41 Mária Street, Budapest, Hungary. \\ ${ }^{3}$ Institute of Medical Microbiology, Semmelweis University, 4 Nagyvárad \\ Square, Budapest, Hungary.}

Competing interests

The authors declare that they have no competing interests and no specific funding was provided for this study.

Availability of data and materials

Presented in the main paper.

Received: 19 February 2016 Accepted: 13 September 2016

Published online: 20 September 2016

References

1. Tapsall JW. Antibiotic resistance in Neisseria gonorrhoeae. Clin Infect Dis. 2005;41:263-8.

2. Bignell C, Unemo M, European STI Guidelines Editorial Board. European guideline on the diagnosis and treatment of Gonorrhoea in adults. Int J STD AIDS. 2013;24:85-92.

3. Brunner A, Nemes-Nikodem E, Mihalik N, Marschalko M, Karpati S, Ostorhazi E. Incidence and antimicrobial susceptibility of Neisseria gonorrhoeae isolates from patients attending the national $N$. gonorrhoeae reference laboratory of Hungary. BMC Infect Dis. 2014;14:433. 
4. Steingrimsson O, Olafsson JH, Thorarinsson H, Ryan RW, Johnson RB, Tilton RC. Azithromycin in the treatment of sexually transmitted disease. J Antimicrob Chemother. 1990;25:109-14.

5. Amrol D. Single-dose azithromycin microsphere formulation: a novel delivery system for antibiotics. Int J Nanomed. 2007;2:9-12.

6. European Centre for Disease Prevention and Control (ECDC). Antimicrobial consumption interactive database (ESAC-Net). 2015. http://www. ecdc.europa.eu/en/healthtopics/antimicrobial_resistance/esac-netdatabase/Pages/database.aspx.

7. Országos Epidemiológiai Központ (OEK)/National Epidemiological Centre. Szexuális úton terjedó betegségek Magyarországon/Sexually transmitted diseases in Hungary 2013. IV. Epinfo. 2014;5:45-50.

8. The European Committee on Antimicrobial Susceptibility Testing. Breakpoint tables for interpretation of MICs and zone diameters. Version 4.0. 2014. http://www.eucast.org.

9. Martin IM, Ison CA, Aanensen DM, Fenton KA, Spratt BG. Rapid sequencebased identification of gonococcal transmission clusters in a large metropolitan area. J Infect Dis. 2004;189:1497-505.

10. Tamura K, Stecher G, Peterson D, Filipski A, Kumar S. MEGA6: molecular evolutionary genetics analysis Version 6.0. Mol Biol Evol. 2013:30:2725-9.

11. http://blast.ncbi.nlm.nih.gov/Blast.cgi?PAGE_TYPE $=$ BlastSearch\&PROG_ DEF=blastn\&BLAST_PROG_DEF=megaBlast\&BLAST_SPEC=blast2seq.

12. Kenyon C, Osbak K. Certain attributes of the sexual ecosystem of high-risk MSM have resulted in an altered microbiome with an enhanced propensity to generate and transmit antibiotic resistance. Med Hypotheses. 2014;83:196-202.

13. Starnino S, Stefanelli P. Neisseria gonorrhoeae Italian Study Group. Azithromycin-resistant N. gonorrhoeae strains recently isolated in Italy. J Antimicrob Chemother. 2009:63:1200-4.
14. Ng LK, Martin I, Liu G, Bryden L. Mutation in 23 S rRNA associated with macrolide resistance in Neisseria gonorrhoeae. Antimicrob Agents Chemother. 2002:46:3020-5.

15. Nemes-Nikodém É, Brunner A, Pintér D, Mihalik N, et al. Antimicrobial susceptibility and genotyping analysis of Hungarian Neisseria gonorrhoeae strains in 2013. Acta Microbiol Immunol Hung. 2014;61:435-45.

16. Chisholm SA, Unemo M, Quaye N, et al. Molecular epidemiological typing within the European gonococcal antimicrobial resistance surveillance programme reveals predominance of a multidrug-resistant clone. Euro Surveill. 2013;18:20358.

17. Palmer HM, Young $\mathrm{H}$, Graham C, Dave J. Prediction of antibiotic resistance using Neisseria gonorrhoeae multi-antigen sequence typing. Sex Transm Infect. 2008;84:280-4.

18. Chisholm SA, Wilson J, Alexander S, Tripodo F, Al-Shahib A, Schaefer $\mathrm{U}$, Lythgow K, Fifer H. An outbreak of high-level azithromycin resistant Neisseria gonorrhoeae in England. Sex Transm Infect. 2015;92:365-7. doi:10.1136/sextrans-2015-052312.

19. Furuya R, Nakayama $H$, Kanayama A, et al. In vitro synergistic effects of double combinations of beta-lactams and azithromycin against clinical isolates of Neisseria gonorrhoeae. J Infect Chemother. 2006;12:172-6.

20. Barbee LA, Soge OO, Holmes KK, Golden MR. In vitro synergy testing of novel antimicrobial combination therapies against Neisseria gonorrhoeae. J Antimicrob Chemother. 2014;69:1572-8.

21. Hauser $C$, Hirzberger L, Unemo M, Furrer $H$, Endimiani A. In vitro activity of fosfomycin alone and in combination with ceftriaxone or azithromycin against clinical Neisseria gonorrhoeae isolates. Antimicrob Agents Chemother. 2015:59:1605-11.

22. Ross JDC, Lewis DA. Cephalosporin resistant Neisseria gonorrhoeae: time to consider gentamicin? Sex Transm Infect. 2012;88:6-8.

23. Lewis DA. Global resistance of Neisseria gonorrhoeae: when theory becomes reality. Curr Opin Infect Dis. 2014;27:62-7.

\section{Submit your next manuscript to BioMed Central and we will help you at every step:}

- We accept pre-submission inquiries

- Our selector tool helps you to find the most relevant journal

- We provide round the clock customer support

- Convenient online submission

- Thorough peer review

- Inclusion in PubMed and all major indexing services

- Maximum visibility for your research

Submit your manuscript at www.biomedcentral.com/submit
O Biomed Central 